\title{
N94-11555
}

\section{A Combustion Products Analyzer for Contingency Use During Thermodegradation Events on Spacecraft}

\author{
Steve Wilson, Thomas F. LImero, Steve W. Beck \\ KRUG Life Sciences Inc., 1290 Hercules Dr., Suite 120, Houston, TX 77058 \\ John T. James \\ Lyndon B. Johnson Space Center, NASA, SD4, Houston, TX 77058
}

\section{ABSTRACT}

The Toxicology Laboratory at Johnson Space Center (JSC) and Exidyne Instrumentation Technologies (EIT) have developed a prototype Combustion Products Analyzer (CPA) to monitor, in real time, combustion products from a thermodegradation event on board spacecraft. The CPA monitors four gases that are the most hazardous compounds (based on the toxicity potential and quantity produced) likely to be released during thermodegradation of synthetic materials: hydrogen fluoride (HF), hydrogen chloride $(\mathrm{HCl})$, hydrogen cyanide $(\mathrm{HCN})$, and carbon monoxide (CO). The levels of these compounds serve as markers to assist toxicologists in determining when the cabin atmosphere is safe for the crew to breathe following the contingency event.

The CPA is a hand-held, battery-operated instrument containing four electrochemical sensors, one for each target gas, and a pump for drawing air across the sensors. The sensors are unique in their small size and zero-g compatibility. The immobilized electrolytes in each sensor permit the instrument to function in space and eliminate the possibility of electrolyte leaks. The sample inlet system is equipped with a particulate filter that prevents clogging from airborne particulate matter. The CPA has a large digital display for gas concentrations and warning signals for low flow and low battery conditions.

The CPA has flown on 13 missions beginning with STS 41 in October, 1990. Current efforts include the development of a microprocessor, an improved carbon monoxide sensor, and a ground-based test program to evaluate the CPA during actual thermodegradation of selected materials.

\section{INTRODUCTION}

The Toxicology Laboratory at NASA Johnson Space Center (JSC) has been directly involved in developing a Combustion Products Analyzer (CPA) to monitor combustion products from a thermodegradation of nonmetallic materials on board spacecraft. The term "thermodegradation," as used in this paper, refers to the full range of events from an overheated electrical component or wire to a major fire. The likelihood of significant thermodegradation events occuring on orbit, as evident by recent incidents on the Space Shuttle, have driven this development effort (1).

The release of potentially toxic gases into the spacecraft atmosphere during a major thermodegradation incident would require the crew to breathe directly from the spacecraft oxygen supply to obtain clean air. Since this oxygen supply is limited, the mission would likely be terminated if the quality of the cabin air could not be varified as safe within a reasonable time. Input from the crew and spacecraft systems as to the magnitude of the event will be very important in the decision-making process. However, thermodegradation of very small quantities of some synthetic materials can produce hazardous levels of decomposition products. Levels of toxic products in the atmosphere would be difficult to assess by observation or symptoms; thus, without real-time monitoring capability, restriction to a conservative decision will lend to an aborted mission. Methods exist for decontaminating the cabin atmosphere of many of the compounds likely to be released from thermodegradation of nonmetallic materials. The CPA has been designed to monitor the effectiveness of these procedures, with the goal of determining when the air is safe for the crew to breathe. 
SHUTTLE EXPERIENCE - During Shuttle missions, five thermodegradation incidents are known to have taken place. Although these incidents were relatively minor, each provided valuable information to the NASA Toxicology Laboratory and reinforced the need for the CPA.

The first thermodegradation event occurred on STS-6 in May 1983 (2). During this mission, the crew sensed a persistent odor emanating from the area of a payload experiment (monodisperse latex reactor). Evaluations after the mission revealed that several kapton-teflon insulated wires beneath the dehumidifier were found to be fused together. Postflight testing of the kapton wire did not demonstrate conclusively that the odor came from the fused wires. The crew did not experience any apparent adverse health effects as a result of this incident.

A second thermodegradation incident was reported on STS-28 in August 1989 (3). On the fifth day of flight, a teleprinter cable located on the flight deck shorted for 1.5 seconds. Postflight analysis indicated that 0.1 gram of Teflon had been pyrolyzed during the incident. Although pyrolysis of this amount of teflon did not pose a hazard to the crew, calculations showed that burning of 2 grams of teflon could have caused adverse health effects in the crew (4).

Other events occurred aboard STS-35 in December 1990 (5). During the first day of flight, the crew detected a burning odor from the Digital Display System 1 (DDS), which automatically shut down. During the fifth day of the mission a second unit, DDS2, also failed, emitting a pungent odor. This unit too shut down automatically. Another attempt to power DDS1 that day also produced a burning odor. Postflight analysis led to the conclusion that clogged air filters caused overheating of several electrical components in both DDS units. Crew health did not appear to be affected as a result of this incident.

The most recent incident occurred during STS-40 when a small motor, containing Delrin (polyformaldehyde) and surrounded by foam, seized and overheated. The result was noxious odors which filled the middeck and caused headache and nausea in some crewmembers (6).

Although these relatively limited thermodegradation incidents to date have not affected crew health or performance, they clearly demonstrate the potential for significant problems.

TARGET COMPOUND SELECTION - The first stage in the development of the CPA was to determine which compounds should be monitored. Many variables affect which products are generated in a thermodegration event, e.g. type of material, temperature, oxygen content, microgravity, etc.. Measuring every toxic compound that is generated during a thermodegradation event is impossible. Toxicologists at NASA identified four compounds that will be used as markers for characterizing the air quality during an incident (7). These compounds are carbon monoxide (CO), hydrogen fluoride (HF), hydrogen cyanide $(\mathrm{HCN})$, and hydrogen chloride $(\mathrm{HCl})$. These are not necessarily the most toxic chemicals generated in such an event. However, considering both toxic properties and relative quantities of thermodegradation products likely generated, these 4 chemicals are considered to be the most hazardous (7). In other words, it is unlikely that any thermodegradation event will generate hazardous levels of any other compound without at least one of these targeted chemicals being present at levels affecting crew health and safety. Consequently, the selected compounds would serve as "markers" during decontamination efforts to indicate when the cabin air was safe to breathe. Potential sources of the targeted compounds in the Space Shuttle are listed in Table I. The spacecraft maximum allowable concentrations (SMACs) (8) and threshold limitvalues (TLVs), set by NASA toxicologists and the American Conference of Governmental Industrial Hygienists (ACGIH) (9), respectively, are listed in Table II.

Table I: Hazardous Thermodegradation

Products From Selected Materials

\begin{tabular}{|c|c|c|c|}
\hline KAPTON & TEFLON & PVC & $\begin{array}{c}\text { POLY- } \\
\text { URETHANE }\end{array}$ \\
\hline $\mathrm{CO}$ & $\mathrm{CO}$ & $\mathrm{CO}$ & $\mathrm{CO}$ \\
$\mathrm{HCN}$ & $\mathrm{HF}$ & $\mathrm{HCl}$ & $\mathrm{HCN}$ \\
& $\mathrm{COF}_{2}$ & & \\
\hline
\end{tabular}

Table II. Exposure Limits (ppm) for Targeted Gases

\begin{tabular}{|c|c|c|c|c|}
\hline & CO & HF & HCN & $\mathrm{HCl}$ \\
\hline TLV & 50 & $* 3$ & $* 10$ & ${ }^{*} 5$ \\
$\begin{array}{c}\text { SMAC } \\
\text { (7-day) }\end{array}$ & 25 & 0.1 & 1 & 1 \\
\hline
\end{tabular}

PROTOTYPEDEVELOPMENT - The next step in the CPA development process was to identify the required and desirable features, and specifications 591 for an analyzer capable of measuring the 4 targeted 
compounds at meaningful concentrations in the spacecraft atmosphere. This would be followed by an investigation of available technologies to meet the defined criteria.

The required or desirable features included the following: simultaneous measurement of all targeted compounds, highly selective, compact, lightweight, microgravity compatible, impervious to the vibration and shock of launch, simple to use, low maintenance requirements, battery-operated, and no spacecraft resource requirements with the exception of power during extended continuous operating periods (on the order of days). Specifications were developed for response and recovery times, signal-to-noise ratios, weight, volume, battery-operation time, accuracy, detection limits, working ranges, calibration requirements, display requirements, and others.

Four technologies, infrared spectroscopy, catalytic surface sensors, electrochemistry, and lengthof-stain indicator tubes, became the focus of the selection effort. Limitations were identified in all the technologies; however, electrochemical sensing met more of the required criteria than any other technology. Companies in the electrochemical sensor industry were identified and contacted. Exidyne Instrumentation Technologies (EIT) of Exton, Pennsylvania, one of only a few companies which manufacture their own electrochemical sensors, was selected to build a prototype combustion products analyzer. The culmination of a 6-month contract with EIT in 1990 resulted in the CPA described below.

The prototype CPA has four electrochemical sensors to monitor $\mathrm{CO}, \mathrm{HF}, \mathrm{HCN}$, and $\mathrm{HCl}$. The sensors are arranged in a teflon block so that the face of each sensor is exposed to a channel through which ambient air is pumped at a rate of $15 \mathrm{~cm} / \mathrm{s}$ (10) (Figure 1). A $25-\mathrm{mm}$ particulate filter prevents entry of particles larger than $0.3 \mu$. The front panel of the CPA is shown in Figure $2(10)$. The entire unit weighs 3 lbs., with a volume of only 77 cubic inches. A battery (recharged

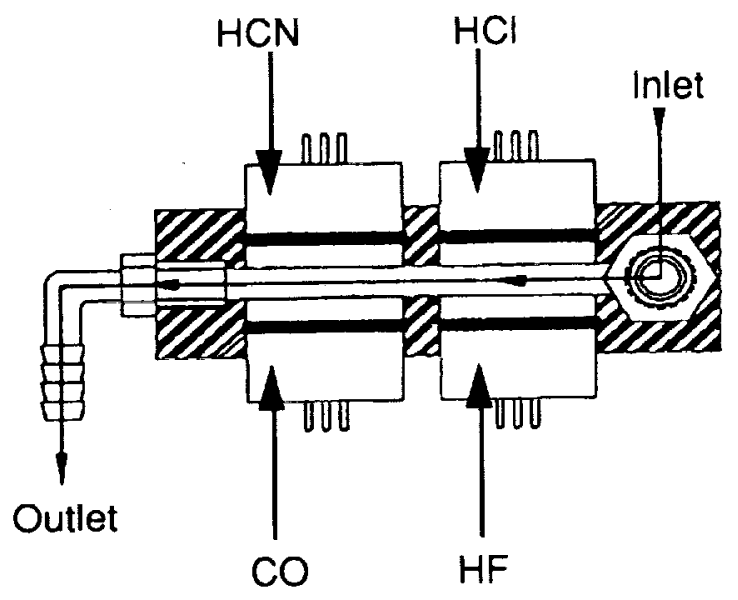

Flgure 1: CPA Sensor Block

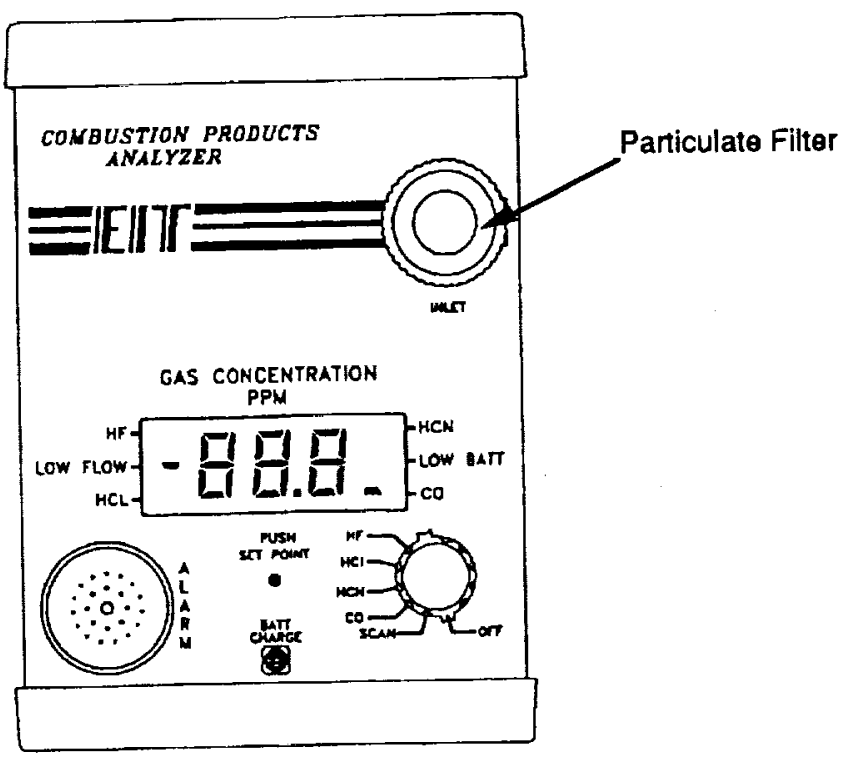

Figure 2: Front Panel of CPA

on 28 VDC power source) provides up to 16 hours of continuous operation. The instrument is capable of operating continuously using 28 VDC spacecraft power. In use, the crew need only switch the instrument from off to the scan mode. In the scan mode, the concentration (ppm) of each compound is displayed sequentially for approximately 5 seconds; however, a single compound can also be selected for continuous monitoring by turning the switch to the appropriate compound position. In order to avoid false readings due to mechanical failures, low-battery and low-flow warnings have been incorporated into this compact instrument. Manual alarm levels can be set to trip an audio alarm when any of the preset values are exceeded. Instrument calibration is easily performed by removing the back panel exposing the zero and span potentiometers for each sensor. The EIT sensors include electronic temperature-compensation capabilities that allow the sensors to operate accurately at temperatures ranging from 5 to $40^{\circ} \mathrm{C}$.

SENSOR DESIGN AND THEORY - Many of the electrochemical sensors commercially available today are relatively large and depend on free-flowing liquid electrolyte. EIT's unique sensor design reduced the electrode size and immobilized the liquid electrolyte, thereby decreasing volume and permitting operation in microgravity. A general schematic of the electrochemical amperometric sensors is shown in Figure $3(10)$. The gas passes through the first membrane, which is designed to provide a quiescent zone for diffusion. The second gas-diffusion membrane and a sensing electrode are bound to each other. Sandwiched between the counter/reference electrode and the sensing electrode is the electrolyte, immobilized in a microfibrous matrix. Selectivity for each sensor may be gained by choice of electrolyte, 


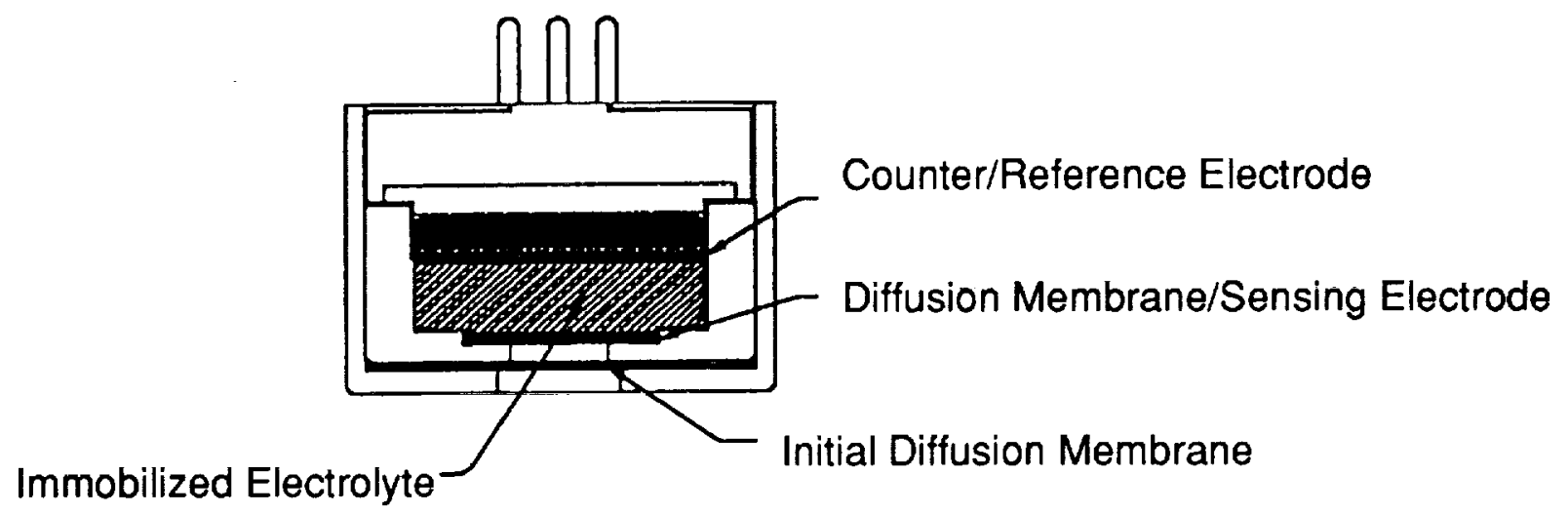

Figure 3: CPA Sensor Schematic

noble metal electrodes, electrode potentials, prefilters, and gas-diffusion membrane materials. Although these sensors can operate by passive air diffusion, a pump was added to ensure adequate response and recovery times.

Each targeted gas diffuses through the membrane and the sensing electrode before reaching the electrode/electrolyte interface, where the electrochemical reactions occur (see Figure 3 ). The sensing electrode and counter/reference electrode provide the closed circuit necessary for the oxidation-reduction reaction. If sensor conditions are optimized, the reactant of interest will react readily at the electrode/ electrolyte interface and the concentration of reactant at the interface will be zero; hence the measured current is proportional to the concentration of the reactant in the bulk gas. Using a modification of Fick's law of diffusion, this can be shown to be a linear relationship (10).

Given the appropriate potential, the $\mathrm{CO}, \mathrm{HCN}$, and $\mathrm{HCl}$ are direct oxidation reactions, whereas the $\mathrm{HF}$ is detected indirectly as an acid gas by a reduction reaction (10).

\section{RESULTS AND DISCUSSION}

SENSOR PERFORMANCE - General operating characteristics of the sensors are presented in Table III. The range, resolution, accuracy, repeatability, and reaction times of the sensors are sufficient for the proposed monitoring functions of the CPA during a thermodegradation event. The response and recovery times for the HF sensor, although adequate, are slower than desirable. The slower times can be attributed to the indirect reactions required to detect this compound. Efforts have begun to improve these response times.

One of the most importantparameters when evaluating an analytical procedure is the degree of specificity of the technique or reaction. A procedure that is subject to significant false positive or false negative reactions is unreliable and useless. Since the purpose of the CPA is to provide valuable information during a contingency situation, a great deal of effort has been involved in identifying chemicals that either positively or negatively interfere with the sensors producing ambiguous results.

Table III. Results of CPA Sensor Performance Testing

\begin{tabular}{|l|c|c|c|c|}
\hline & HCI & HF & HCN & CO \\
\hline RANGE & $0-99.9$ PPM & $0-99.9$ PPM & $0-99.9$ PPM & $0-999$ PPM \\
RESOLUTION & $0.1 \mathrm{PPM}$ & $0.1 \mathrm{PPM}$ & $0.1 \mathrm{PPM}$ & $1 \mathrm{PPM}$ \\
ACCURACY* & $\pm 5 \%$ & $\pm 5 \%$ & $\pm 5 \%$ & $\pm 2 \%$ \\
REPEATABILITY & $\pm 2 \%$ & $\pm 2 \%$ & $\pm 2 \%$ & $\pm 1 \%$ \\
RESPONSE TIME & & & & \\
RISE TO 80\% & 2 minutes & 5 minutes & 1 minute & 15 seconds \\
DECAY TO 90\% & 2 minutes & 8 minutes & 1 minute & 30 seconds \\
\hline
\end{tabular}


Table IV. Results of Cross-Sensitlvity Testing of CPA Sensors

\begin{tabular}{|c|c|c|c|c|c|}
\hline GAS & CONCENTRATION & HCl & HF & HCN & CO \\
\hline CO & 50 PPM & 0 & 0 & 0 & 50 \\
$\mathrm{HCl}$ & $6 \mathrm{PPM}$ & 6 & 4.0 & 0 & 0 \\
$\mathrm{HF}$ & $5 \mathrm{PPM}$ & 0 & 5.0 & 0 & 0 \\
$\mathrm{HCN}$ & $42 \mathrm{PPM}$ & 0.4 & -5.7 & 42 & 7 \\
\hline
\end{tabular}

Cross-sensitivity reactions within the four gases are shown in Table IV (10). The HCN sensor exhibits no significant cross-sensitivity when exposed to the other targeted gases. The CO sensor shows a crosssensitivity to HCN gas in a ratio of approximately $6: 1-$ meaning $6 \mathrm{ppm}$ HCN produces a signal in the $\mathrm{CO}$ sensor equal to $1 \mathrm{ppm} \mathrm{CO}$. The $\mathrm{HF}$ and $\mathrm{HCl}$ sensors, however, demonstrate cross-sensitivities that are related to their general response to the acid moiety. These cross-sensitivity reactions are predictable and can be easily corrected using a microprocessor, with fully-compensated data being displayed to the user. Development of a microprocessor has begun and will be discussed further below.

Additionally, the sensors have been exposed to numerous other gases in an effort to identify compounds that can act as interferents. Most of the gases that interfere (ozone, ammonia, sulfur dioxide, fluorine, bromine, chlorine, methyl sulfide, and hydrogen sulfide) either will not be present on spacecraft, exist at extremely low concentrations, or produce an insignificant cross-sensitivity response. The sensors do not respond to other potential interferents (carbon dioxide, ethanol, and methane) that are known to be present in the Shuttle atmosphere in significant quantities.

MISSION RESULTS - Beginning with STS-41 in October 1990, the CPA has flown on all missions, totalling 13 to date. Typical on-orbit use of the CPA involves the collection of baseline readings each day of the mission. Results to date indicate that the sensors have performed nominally on orbit and have remained in calibration during the course of the mission with the exception of a problem with the $\mathrm{CO}$ sensor discussed below. The daily readings of the $\mathrm{HCl}, \mathrm{HCN}$, and $\mathrm{HF}$ sensors have remained very low during these missions, indicating that cross-reacting chemicals that might interfere are not present in significant quantities in the Shuttle atmosphere during nominal operations. Sensor performance is apparently not affected by lift-off or microgravity operation. The average and standard deviation of $\mathrm{HCl}, \mathrm{HCN}$, and
HF sensor readings for all of the missions is presented in Table V.

A significant problem with the $\mathrm{CO}$ sensor was identified very early in the flight test program. Analyses of grab samples collected during the course of the missions found much lower $\mathrm{CO}$ levels than those recorded on orbit by the CPA. Although not a routine analysis at the time, hydrogen was found in the grab samples at levels significant for cross-sensitivity considerations. Subsequent testing of the CPA determined that exposing the $\mathrm{CO}$ sensor to a hydrogen concentration of $100 \mathrm{ppm}$ produces a response of approximately $50 \mathrm{ppm}$ in the $\mathrm{CO}$ sensor (2:1 crosssensitivity ratio). Furthermore, the $C O$ sansor reading exhibited a slow drift upward the lor.jer the sensor was exposed to the hydrogen.

Table V. Mission Data for $\mathrm{HCl}, \mathrm{HF}$, and HCN Sensors

\begin{tabular}{|c|c|c|}
\hline & $\begin{array}{c}\text { Avg. Response } \\
(\mathrm{ppm})\end{array}$ & Std. Dev. \\
\hline $\mathrm{HCl}$ & 0.4 & 0.35 \\
$\mathrm{HF}$ & 0.4 & 0.40 \\
$\mathrm{HCN}$ & 0.1 & 0.1 \\
\hline \multicolumn{2}{|c|}{$\mathrm{n}=128$} \\
\hline
\end{tabular}

EIT and the JSC Toxicology Laboratory embarked on a program to modify the $\mathrm{CO}$ sensor to reduce the cross-sensitivity to hydrogen and to stabilize this response. Considerable improvements were made in the cross-sensitivity ratio (approximately 10:1) and response stability to hydrogen by elevating the voltage bias of the sensing electrode with respect to the reference electrode in the electrochemical cell. The - polarization of the sensing electrode with respect to the reference electrode resulted in a larger zero offset but was not detrimental to the signal-to-noise ratio. The modifications have resulted in a CPA CO sensor 
that has a much improved cross-sensitivity to hydrogen and responds to hydrogen in a predictable manner. The modified sensor has flown on all missions beginning with STS-37 in April 1991. These improvements in the $\mathrm{CO}$ sensor greatly increase its value as a trend indicator in the event of a thermodegradation event.

\section{CURRENT AND FUTURE EFFORTS}

CURRENT EFFORTS - The focus of current development efforts are in two main areas-1) further improvements in the cross-sensitivity problems of the CPA and 2) ground-based testing of the CPA in atmospheres containing combustion/pyrolysis products from the thermodegradation of various nonmetallic materials used on the Orbiter.

Current contractual work with EIT is scheduled for completion in the early fall, 1992. The focus of this work is 1) the fabrication and testing of a hydrogen sensor to be used in conjunction with the CO sensor, and 2) the fabrication and testing of a microprocessor capable of compensating outputs for intra-sensor cross-sensitivities. The hydrogen sensor has been built and is currently being tested for long-term stability. The addition of a fifth sensor will require a larger sensor block; however, this will not substantially increase the size of the instrument. An accurate $\mathrm{CO}$ concentration will be displayed by having a compensation algorithm in the microprocessor. Using the $\mathrm{CO}$ sensor and hydrogen sensor signals and knowing the $\mathrm{CO}: \mathrm{H}_{2}$ cross-sensitivity ratio, corrected data are easily obtained. Alternatively, EIT is exploring ways to reduce the hydrogen interference of the $\mathrm{CO}$ sensor to greater than 100:1. The maximum hydrogen level that has been obtained on any mission is in the 300 ppm range. If this approach is successful, the subtraction procedure would be unnecessary. The microprocessor will also be used to correct the $\mathrm{HCl}$ and HF for their cross-sensitivities to each other and to HCN. These responses are predictable and compensation is easy and straight forward.

In addition to correcting sensor output data, the microprocessor will provide valuable sensor diagnostic information. When the CPA is turned on, the microprocessor will perform a self-check routine. The microprocessor will electronically pulse each sensor to determine if open circuits exist and monitor the resultant current generated by the sensors for their characteristic output signatures. Sensors that are behaving erratically or severely out of calibration will exhibit an uncharacteristic output signature. If a problem is diagnosed, a display will indicate which sensor is not functioning properly.

The other major ongoing effort is a test program being conducted at the NASA White Sands Test Facility (WSTF) to evaluate the CPA's response to combustion products generated from the thermal decomposition of polymeric materials in air (11). This program is in progress and should be completed by January 1993. The materials to be tested include Kapton (fluorocarbon/polyimide wire insulation), pyrell foam (polyurethane foam), polyvinyl chloride (unplasticized PVC polymer), tefzel (ethylenetetrafluoroethylene copolymer wire insulation), and an electronic circuit board with components (glass/ epoxy laminate with capacitors, resistor, and diode). Although the testing will be conducted under defined conditions in a $1-g$ environment, the information gathered should be extremely valuable for further definition of sensors needing improvement and limitations of the hardware. Furthermore, successful operation of the CPA in the combustion atmospheres will provide confidence to the crew, toxicologists, etc., that this instrument will provide useful information during an actual combustion event.

\section{FUTURE ENDEAVORS}

The next step in the development of operational CPA for Shuttle is the inclusion of the hydrogen sensor and microprocessor into an integrated flight hardware package. If modifications are required, based on findings from the WSTF testing, they would be made during this step.

An important component of the Crew Health Care System (CHeCs) in the Space Station Freedom (SSF) Program is the Compound Specific Analyzer-Combustion Products, known as the CSA-CP. The CSA$\mathrm{CP}$ will be a modified CPA. Major modifications will include the addition of data logging capability, interfacing with the SSF data management and power distribution systems, and on-orbit calibration capability.

Keeping a sensitive instrument calibrated during extended missions is a major concern. In its current configuration, the CPA would require calibration approximately every 60 to 90 days using $\mathrm{HCl}, \mathrm{HCN}, \mathrm{HF}$, and $\mathrm{CO}$ gases and this, clearly, could not be performed on orbit. The Toxicology Laboratory and EIT are working on a unique alternative to the standard calibration method. An entire sensor block, containing all four sensors, and an amplifier and microprocessor for each sensor, will be replaced on a regular basis. The microprocessor will store the calibration curves, which will be developed during ground tests; therefore, replacement of the sensor pack will recalibrate the CSA-CP. Multiple sensor packs, enough to support an extended mission, would be 595 calibrated at the same time, sealed, and stored on the 
spacecraft. Sufficient numbers of compact sensor packs could easily be manifested to accommodate extended missions.

The development of a data logger is also being pursued to assist in trend analysis and to provide a record of decontamination effectiveness following a thermodegradation incident. This data logger would also supply information necessary for trend analysis, such as time-weighted averages and coefficients of variance.

\section{CONCLUSIONS}

The Toxicology Laboratory at Johnson Space Center identified a need to monitor toxic combustion products during a thermodegradation event on board spacecraft. A development effort with Exidyne Instrumentation Technologies culminated in the fabrication of the Combustion Products Analyzer, capable of measuring 4 targeted gases. Through successful laboratory and flight test programs, some problems were identified, and modifications are ongoing to solve these problems. Ground-based combustion tests with the CPA using selected spacecraft materials have begun. Several other improvements aimed at upgrading the CPA to meet SSF program requirements have also begun.

\section{REFERENCES}

1. Limero, Thomas; James, John; Cromer, Raymond; Beck, Steve, "A Combustion Products Analyzer for Contingency Use During Thermodegradation Events on Spacecraft", International Conference on Environmental Systems, San Francisco, California, July, 1991.

2. Huntoon, Carolyn, "STS-6 Toxicology Mission Report", NASA- Johnson Space Center memorandum SD4/82-215, 1982.

3. Huntoon, Carolyn, "STS -28 Toxicology Mission Report", NASA- Johnson Space Center memorandum SD4/89-316, 1989.

4. Communications with Drs. John James, NASA-JSC, King-Lit Wong, Hector Garcia, and Chiu-Wing Lam, KRUG Life Sciences, Inc., August, 1989.

5. Huntoon, Carolyn, "STS-35 Toxicology Mission Report", NASA- Johnson Space Center memorandum SD4/91-027, 1991.
6. Huntoon, Carolyn, "STS-40 Toxicology Mission Report", NASA- Johnson Space Center memorandum SD4/91-362, 1991.

7. Communications with Drs. John James, NASA-JSC, King-Lit Wong, Hector Garcia, and Chiu-Wing Lam, KRUG Life Sciences, Inc., various periods in 1989.

8. "Spacecraft Maximum Allowable Concentrations for Airborne Contaminants", Medical Sciences Division, Biomedical Operations and Research Branch, NASA-Johnson Space Center, May, 1990.

9. "1991-1992 Threshold Limit Values for Chemical Substances and Physical Agents and Biological Exposure Indices", The American Conference of Governmental Industrial Hygienists, Cincinnati, $\mathrm{OH}, 1991$.

10. Cromer, Raymond, "Combustion Products Analyzer (CPA) Development: Final Report", KRUG Life Sciences Contract \# 13652 November, 1990.

11. "Test Plan - Evaluation of EIT Combustion Products Analyzer", NASA-White Sands Test Facility, Document No. TP-WSTF-639, December, 1991. 